\title{
SURVEYS FOR FIELD CARBON STARS IN THE MAGELLANIC CLOUDS
}

\author{
M. AZZOPARDI ${ }^{1}$, E. REBEIROT ${ }^{1}$ and B.E. WESTERLUND ${ }^{2}$ \\ ${ }^{1}$ Observatoire de Marseille, 2 Place Le Verrier, F-13248 Marseille Cedex 4, France \\ ${ }^{2}$ Astronomical Observatory, Uppsala University, Box 515, S-751 Uppsala, Sweden
}

The Asymptotic Giant Branch (AGB) stage is the normal luminous phase of evolution of intermediate-mass stars (slightly less than $1 M \odot$ up to very roughly $3 M \odot$ ) and therefore the AGB stars play a noteworthy role in our understanding of the chemical evolution of galactic systems. Furthermore it is now generally accepted that the ratio of carbon (C) stars to late-type $M$ giant stars can be used to estimate the mean metallicity of the stellar population of a system, or the change in metallicity from the inner to the outer parts of a galaxy. It is for these reasons among others that red giant stars have been searched for in nearby local group galaxies, principally in our nearest neighbours, the Magellanic Clouds and the dwarf spheroidal galaxies. This paper will be particularly devoted to the slitless spectroscopy surveys for carbon stars in the Magellanic Clouds.

The detection of carbon stars is made possible, in even relatively distant external galaxies, by the presence in their spectra of prominent molecular absorption bands. In the near-infrared spectral region, carbon stars can be identified mainly through the $\mathrm{CN}$ bands at 7945, 8125 and 8320 A (see Nassau \& Velghe 1964). Sanduleak \& Philip (1977) showed that carbon stars can also be efficiently detected in the blue-green spectral domain through the strong Swan $\mathrm{C}_{2}$ bands at 4737 and $5165 \AA$ (also the $\lambda 5636 C_{2}$ band, when available). Obviously, the surveys based on the recognition of the $\mathrm{CN}$ bands favour the detection of the reddest carbon stars, namely the classic $\mathrm{N}$-type stars, while those based on the identification of the $\mathrm{C}_{2}$ bands allow the detection of the bluer ones, as previously discussed by Westerlund et al. (1986), McCarthy (1987), Blanco \& McCarthy (1990). Owing to their circumstellar dust shells due to strong stellar winds, the most luminous carbon stars can only be detected thanks to infrared or radio observations.

Early surveys for field carbon stars in the Magellanic Clouds were carried out by Westerlund in the late 1950s at Mount Stromlo Observatory using the Uppsala Southern Station 50/65/173 cm Schmidt telescope equipped with an objective-prism yielding a dispersion of $2100 \AA / \mathrm{mm}$ at the atmospheric A band, and a Schott RG 5 filter. Exposures of 90 minutes duration on hypersensitized Kodak I-N plates allowed him to reach stars as faint as $I \sim 13$ mag resulting in the identification of 302 probable carbon stars (Westerlund et al. 1978) in the Large Magellanic Cloud (LMC). However, the limiting I magnitude achieved was inadequate to allow the detection of carbon stars in the Small Magellanic Cloud (SMC) due to their relative faintness. In the meantime, Sanduleak \& Philip (1977) found 474 carbon stars in the LMC on nitrogen-baked Kodak IIIa-J plates, 60 minute exposures, taken with the Cerro Tololo Inter-American 
Observatory (CTIO) $61 / 91 / 210 \mathrm{~cm}$ Curtis Schmidt telescope equipped with a prism providing $1360 \AA / \mathrm{mm}$ at $\mathrm{H} \gamma$ (plate limit $V$ - 16). Comparison of the Westerlund's carbon star list with theirs led Sanduleak and Philip to the conclusion that "the two surveys probably complement one another and together provide for a high degree of completeness to a limit of about $V \sim 16.5$ or $M_{V} \sim-2$.

Later on, Blanco et al (1980) used a near-infrared transmitting grating (2350 $\AA / m m$ dispersion at the atmospheric $A$ band) and hypersensitized Kodak IV-N plates in combination with a Schott RG 630 filter at the prime focus of the CTIO $4 \mathrm{~m}$ telescope (limiting magnitude $I \sim 17$ obtained in 120 minute exposure) to survey $C$ and late $M$-type stars in the Magellanic Clouds. They found 186 and 134 carbon stars in $3 \mathrm{LMC}$ and 2 SMC fields of $0.12 \mathrm{deg}^{2}$ each. Additional observations of $49 \mathrm{LMC}$ and 35 SMC sample areas resulted in the identification of 1045 field carbon star candidates in the LMC, and 860 in the SMC, on the whole. On account of their adequate degree of completeness, those surveys allowed Blanco \& McCarthy (1983) to infer statistically the surface distribution of the cool carbon stars in the Magellanic Clouds, and to estimate the total carbon-star population of the LMC and SMC to be 11,000 and 2,900, respectively. Subsequently, finding charts and coordinates for 849 cool-carbon LMC stars have been presented by Blanco \& McCarthy (1990).

From 1981 to 1984 a new spectroscopy survey for field carbon stars in the Magellanic Clouds was carried out by Westerlund and associates, adopting the search methods of Sanduleak \& Philip (1977). For this purpose, they used, at the prime focus of the ESO $3.6 \mathrm{~m}$ telescope, a wide field adaptor equipped with a grism giving $2200 \AA / \mathrm{mm}$ dispersion. In order to minimise problems from over-crowding, they restricted the instrumental spectral range to $4350-5300 \AA$ by combining a IIIa-J emulsion with a Schott GG435 filter. 8 circular fields of $0.78 \mathrm{deg}^{2}$ each, located in selected regions of the LMC and 13 partially overlapping fields, which together cover the central regions of the SMC, have been observed. While the survey of the LMC fields is still in progress, the SMC observing material (grism plates as well as subsequent slit spectroscopy and JHK photometry of several carbon star candidates) is now fully reduced. This survey resulted in a catalogue of 1707 SMC field carbon stars (Rebeirot et al. 1993) providing identifications and spectrophotometry. The spectrophotometric quantities (magnitudes, colour-equivalents and carbon abundances) have been obtained from the microdensitometer-scanned grism spectra, using the technique perfected by Westerlund et al. (1986). A number of results have already been inferred from those data. For instance, our more complete star counts (Rebeirot et al. 1987) confirm, on the whole, the overall surface distribution derived from sample areas by Blanco \& McCarthy (1983). Consequently our estimated total number of SMC carbon stars (Azzopardi \& Rebeirot 1991), although slightly larger, is nevertheless, comparable to that found by these authors. The use of the grism spectrophotometric data, namely the relation between the carbon-abundance and the colour equivalent, allowed us to discriminate from the 'normal' carbon stars both the carbonpoor C stars and the J-type stars (Westerlund et al. 1986; Rebeirot et al. 1993). Another important result inferred from our spectrophotometry and subsequent infrared photometry is the discovery of SMC carbon stars at higher temperature and lower luminosity than previously known (Westerlund et al. 1991, 1992). The $M_{b o l}$ luminosity function for 155 SMC carbon stars, for which we have $J H K$ measurements, displayed by Azzopardi (1993), shows that the least luminous carbon stars ever found in an extragalactic system overlap with the most luminous ones that we have detected in the galactic bulge (Westerlund et al. 1991).

Very deep surveys are now in progress (Muratorio \& Azzopardi 1993) to search for intrinsically very faint carbon stars that appear to form, in the HR-diagram, a natural continuation 


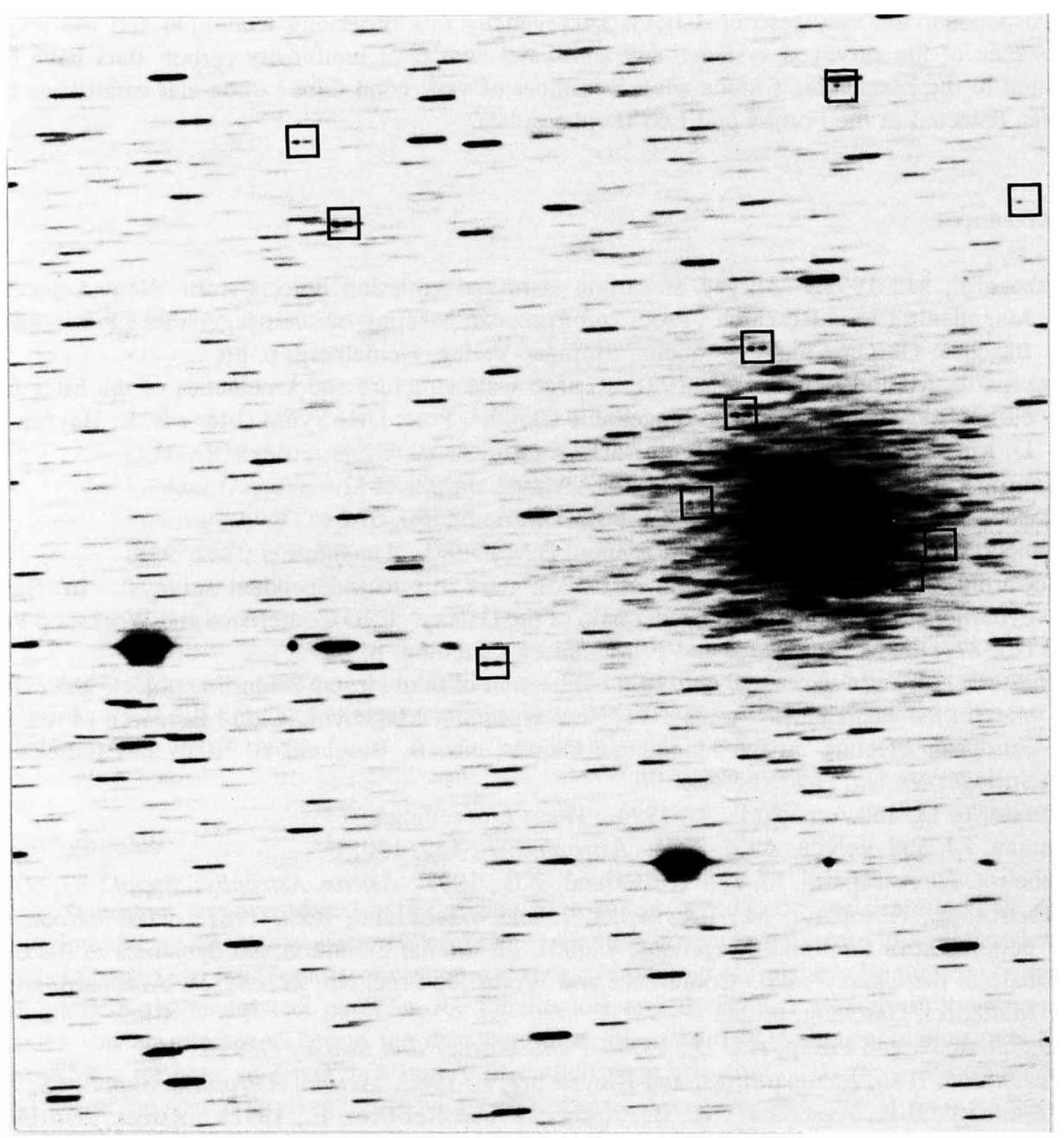

Figure 1. SMC field near NGC 419. Part of a 1 hour exposure ESO $3.6 \mathrm{~m}$ telescope grism plate obtained on IIIa-J emulsion through a Schott GG435 filter. The spectra of the identified carbon stars are marked with squares.

of the galactic-bulge carbon stars towards the more luminous objects although their origin may not be the same. For this purpose we have now undertaken to carry out slitless spectroscopy surveys in selected areas of the Magellanic Clouds and the galactic halo dwarf spheroidal galaxies using the ESO Faint Object Spectrograph and Camera (EFOSC) devices attached to the Cassegrain foci of either the ESO $3.6 \mathrm{~m}$ or the Max Plank Gesellschaft/ESO $2.2 \mathrm{~m}$ telescopes. CCD grism spectroscopy frames are secured through a suitable interference filter (see Muratorio \& Azzopardi, these proceedings) to survey for carbon stars 3 to 4 magnitudes fainter than the grism photographic surveys, while keeping the number of overlaps as low as possible. Taking 
into account the very restricted fields surveyed by this observing technique and the relative distance of the surveyed systems, any additional new faint luminosity carbon stars have been found in the Magellanic Clouds while a number of very good faint carbon star candidates have been detected in the Fornax and Leo II spheroidals.

\section{References}

Azzopardi, M., 1993. 'Survey of carbon stars and emission objects'. In 'New Aspects of Magellanic Cloud Research', Proc. 2nd European Meeting on the Magellanic Clouds, eds B. Baschek, G. Klare and J. Lequeux, Springer-Verlag, Heidelberg, p. 86.

Azzopardi, M. and Rebeirot, E., 1991. 'Large scale structure and kinematics of the MCs from carbon star studies'. In 'The Magellanic Clouds', Proc. IAU Symp. 148, eds. R. Haynes and D. Milne, Kluwer Academic Publishers, Dordrecht, p. 71.

Blanco, V.M. and McCarthy, M.F., 1983. Astron. J., 88, 1442.

Blanco, V.M. and McCarthy, M.F., 1990. Astron. J., 100, 674.

Blanco, V.M., McCarthy, M.F. and Blanco, B.M., 1980. Astrophys. J., 242, 938.

McCarthy, M.F., 1987. 'Detection of carbon stars in two independent surveys'. In 'Stellar evolution and dynamics in the outer halo of the Galaxy', ESO Conference and Workshop Proc. No. 27, eds. M. Azzopardi and F. Matteucci, Garching, p. 203.

Muratorio, M. and Azzopardi, A., 1993. 'Detection of faint $\mathrm{H} \alpha$ emission-line objects and carbon stars in the Magellanic Clouds', In 'New aspects of Magellanic Cloud Research', Proc. 2nd European Meeting on the Magellanic Clouds, eds. B. Baschek, G. Klare and J. Lequeux, Springer-Verlag, Heidelberg, p. 96.

Muratorio, M. and Azzopardi, A., 1994. These proceedings.

Nassau, J.J. and Velghe, A.G., 1964. Astrophys. J., 139, 190.

Rebeirot, E., Azzopardi, M. and Westerlund, B.E., 1993. Astron. Astrophys. Suppl., 97, 603.

Rebeirot, E., Azzopardi, M., Breysacher, J. and Westerlund, B.E., 1987. 'The carbon star population of the Small Magellanic Cloud'. In 'Stellar evolution and dynamics in the outer halo of the Galaxy', ESO Conference and Workshop Proc. No. 27, eds. M. Azzopardi and F. Matteucci, Garching, p. 263.

Sanduleak, N. and Philip, A.G.D., 1977. Publ. Warner and Swasey Obs., 2, 105.

Westerlund, B.E., Azzopardi, M. and Breysacher, J., 1986. Astron. Astrophys. Suppl., 65, 79.

Westerlund, B.E., Azzopardi, M., Breysacher, J. and Rebeirot, E., 1992a. Astron. Astrophys. Suppl., 91, 425.

Westerlund, B.E., Azzopardi, M., Breysacher, J. and Rebeirot, E., 1992b. Astron. Astrophys., 260, L4-6.

Westerlund, B.E., Lequeux, J., Azzopardi, M. and Rebeirot, E., 1991. Astron. Astrophys., 244, 367.

Westerlund, B.E., Olander, N., Richer, H.B. and Crabtree, D.R., 1978. Astron. Astrophys. Suppl., 31, 61. 